\title{
Sinonasal morbidity following tumour resection with and without nasoseptal flap reconstruction*
}

\author{
Richard J. Harvey ${ }^{1,3}$, Joanne Malek', Mark Winder ${ }^{1,2}$, Andrew Davidson³, \\ Tim Steel', Nadine Mrad', Henry Barham', Anna Knisely', Charles Teo ${ }^{4}$ \\ 'Rhinology and Skull Base Research Group, St Vincent's Centre for Applied Medical Research, University of New South Wales, \\ Sydney, Australia \\ 2 Department of Neurosurgery, St Vincent's Hospital, Sydney, Australia \\ ${ }^{3}$ Australian School of Advanced Medicine, Macquarie University, Sydney, Australia \\ ${ }^{4}$ Centre for Minimally Invasive Neurosurgery, Prince of Wales Private Hospital, Sydney, Australia
}

Rhinology 53: 122-128, 2015

DOl:10.4193/Rhino 14.247

*Received for publication:

October 6, 2014

Accepted: November 11, 2014

\begin{abstract}
Background: Sinonasal function can be affected by multiple treatment modalities but surgical techniques, such as the nasoseptal flap or Draf 3 procedure, have been implicated in poor post-treatment function. Prior studies have rarely used comparable populations and this study aims to assess the impact of surgical technique, mainly the nasoseptal flap, on sinonasal function in a group of comparable patients.
\end{abstract}

Methods: A prospective cohort of patients undergoing endoscopic surgery for sinonasal and skull base tumours was studied. Patients were analysed according to whether a nasoseptal flap was used. Other treatment factors included; use of the Draf 3, radiotherapy, removal of olfactory apparatus and dural resection. The Sinonasal Outcome Test 22 (SNOT22), a nasal symptom score (NSS), global function score and nasal obstruction scores were recorded pre and post treatment.

Results: One hundred and eighteen patients were assessed. Forty-two patients had a nasoseptal flap. Perioperative radiotherapy was higher in the nasoseptal group, as was dural resection and the need to remove the olfactory apparatus. Despite this, there was no significant difference in SNOT22 scores and NSS. Radiotherapy was detrimental to sinonasal function with SNOT22 and NSS.

Conclusion: The use of a nasospetal flap in surgery does not affect patient quality of life and sinonasal function after endoscopic tumour resection. Pathology is a better predictor of morbidity, with loss of function from radiotherapy or resection of functional areas such as the olfactory apparatus having a greater impact.

Keywords: Quality of life, skull base, sinonasal, function, obstruction, nasoseptal, septal, flap, neoplasia, tumour

\section{Introduction}

The nasoseptal flap along with other local mucosal flaps, as part of skull base reconstruction, have dramatically changed the reconstructive outcomes for patients with endoscopic endonasal skull base surgery ${ }^{(1)}$. They provide a robust barrier when reconstructing dural defects ${ }^{(2)}$ and are superior to free grafts ${ }^{(1)}$. There are other components of reconstruction, such as collagen inlays that have avoided the donor site morbidity associated with fat or fascia from areas such as abdomen or lateral thigh ${ }^{(3)}$.
Although not necessarily the standard of $\operatorname{care}^{(4)}$, the nasoseptal flap is incredibly valuable as an option for the skull base surgeon when performing endoscopic tumour resections. The nasoseptal flap is not exclusively used to repair dural defects. It can be used to cover important neurovascular structures, such as the internal carotid artery, or provide rapid mucosalization to large areas of exposed bone or when early radiotherapy is required(5). However, concerns have been raised over the impact of the nasoseptal flap on sinonasal function ${ }^{(6,7)}$. The published studies 
investigating the impact of the nasoseptal flap are inherently biased as many include pituitary patients having simple-transphenoidal operations together with patients having more invasive or expanded approaches. They are often not comparable groups. Such comparisons are inherently flawed, as intracranial pathologies are more likely to involve more extensive surgery with greater modification of normal sinuses than simple sellar or extracranial surgeries in these series.

Additionally, function, such as olfaction, may be electively sacrificed in certain intracranial pathologies if the posterior cribriform plate is being traversed but the decision to approach endonasally may represent a less morbid option given the alternative morbidity related to frontal lobe retraction ${ }^{(8)}$. This study aims to assess the symptoms and disease specific quality of life in patients undergoing endoscopic endonasal resection for sinonasal and skull base tumors, some, but not all of whom had a nasoseptal flap. The comparison of two groups, both with large areas of exposed sinus and skull base achieves equipoise more effectively when assessing the potential functional and quality of life impact of the nasoseptal flap.

\section{Materials and methods}

This study was approved by the Hospital Human Research Ethics Committee (SVH09/083). Written informed consent was obtained from all patients.

\section{Population}

Consecutive patients undergoing endoscopic endonasal surgery for tumours of the nose, sinus and skull base were selected, regardless of whether a nasoseptal flap was utilized or not. Patients with pituitary adenomas and simple sella based pathology were excluded. Patients with active chronic inflammatory rhinosinusitis, allergic rhinitis, recreational nasal drug use, any regular nasal medication or a prior history of an airway disorder were excluded. The intervention group was those who had a nasoseptal flap used (for any reason) as part of the tumour resection. The comparison group were those who had an endoscopic resection (intra or extradural) where no nasoseptal flap was harvested. The rationale for nasoseptal flap use was heterogeneous and thus potential bias between the two groups was explored via the confounding factors below. A study period from 2009-2012 was taken to ensure adequate time to last follow-up.

\section{Confounding factors}

Five factors were recorded to assess whether the two groups were comparable. The extent of sinus modification was defined by those patients who had a Draf3 or modified endoscopic Lothrop as part of their surgery (yes/no) ${ }^{(9)}$ and the impact of a true skull base reconstruction where intradural surgery was defined by the resection of dura (yes/no) The influence of the

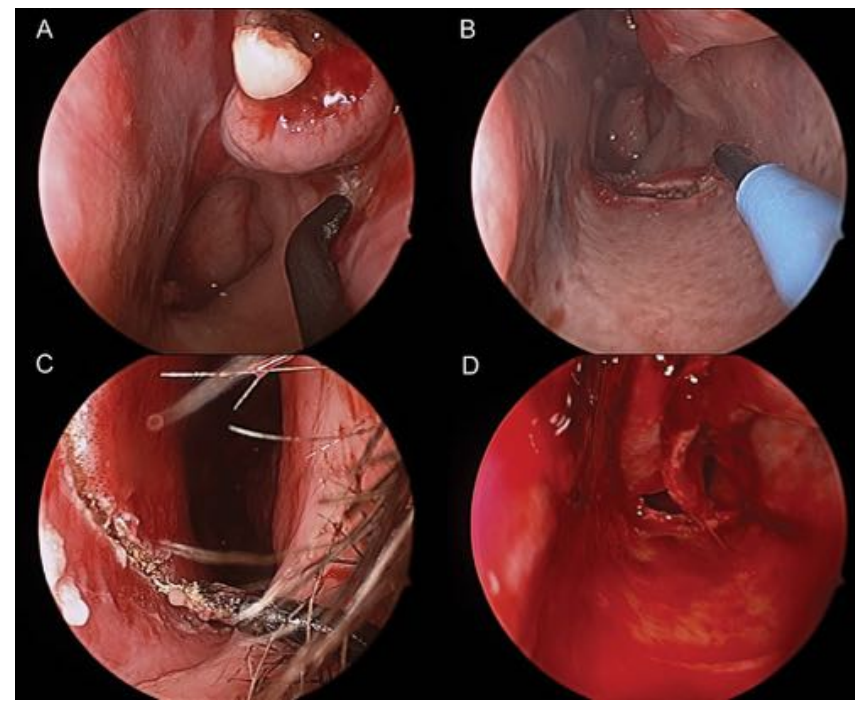

Figure 1. The standard approach to nasoseptal flap when performed as part of extensive tumour surgery. There are many reasons why it is used such as skull base reconstruction, or for coverage of exposed bone or neurovascular structures in paranasal sinus surgery. A flap is not utilised if none of these indications exists, or when the septum is involved by tumor. The pedicle is mobilised to the spehnopalatine foramen with a releasing incision on the medial pterygoid plate $(A)$.The nasal floor is often a considerable part of the flap (B) and raising the flap to the squamo-mucosal junction gains maximum length $(C)$. The final flap being mobilized to the nasopharynx with donor defect (D).

intentional resection of key functional areas was defined as whether the olfactory apparatus was intentionally removed as part of the tumour surgery (yes/no). The impact of adjuvant therapies was defined by having had post-operative radiotherapy as this factor has been associated with worsening of sinonasal function ${ }^{(10,11)}$. Finally, the nature of the pathology (benign/malignant) was used as an overall indicator of therapy as this takes into account several of the above mentioned factors (extent of resection, sacrifice of normal structures, removal of dura and radiotherapy).

\section{Patient reported outcome measures}

Four different constructs of patient reported outcome measures (PROMs) were reported. The sino-nasal outcome 22 test (SNOT22) was used to assess overall disease specific quality of life. This is a validated 22 question survey with four domains: psychological function, sleep function, rhinological symptoms, and ear and/or facial symptoms ${ }^{(12)}$.The SNOT22 is reported as mean of the 22 questions with a score of 0 to 5 . A global rating of sinonasal function on a ordinal scale from -6 (terrible) to 0 (neither good or bad) to +6 (excellent) was also obtained. Nasal symptoms were recorded via a 5 questionnaire Nasal Symptoms score (NSS) from 'nasal obstruction', 'thick nasal discharge,' 'facial 


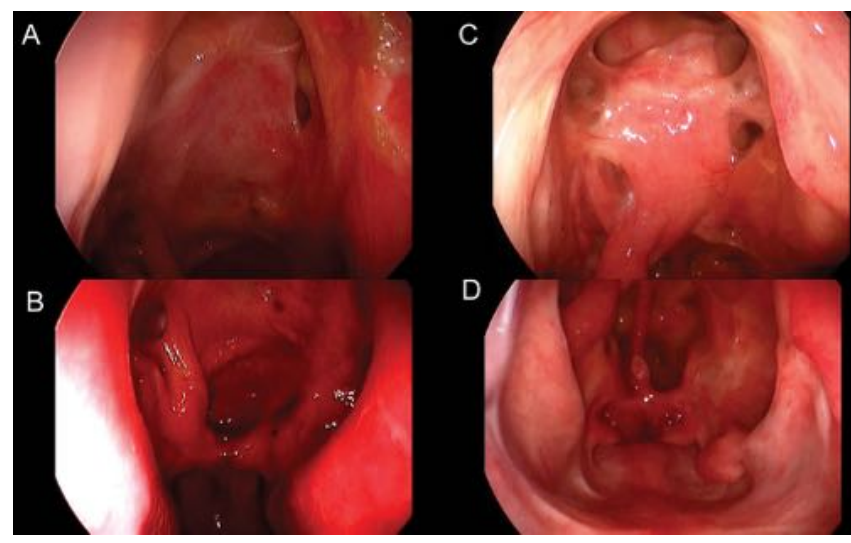

Figure 2. Examples of post radiation post-surgical cavities. A typical neuroblastoma post-surgery and radiotherapy ( $A$ and $B$ ) and a squamous cell carcinoma post-surgery and radiotherapy (C and D). Chronic sinonasal inflammation and crusting, regardless of nasoseptal flap use, are not expected longterm outcomes.

pain/pressure,' 'smell disturbance' and 'need to blow nose'. This was reported as a mean score from 0 to 5 . Nasal obstruction was recorded as a 6 point Likert score from 0 (no problem) to 5 (problem as bad as it could be) ${ }^{(5)}$. All four PROMs were recorded at baseline and at last follow-up.

\section{Surgical technique}

A binostril approach with some form of posterior septal window was the standard approach for most cases in this study. To raise the nasoseptal flap, a medium length needle point monopolar diathermy (Megadyne E-Z Clean 0016AM, Draper, UH, USA) was used on settings of 12 cut and 12 coagulate power (ForceFX 8CS, Valleylab, Boulder, CO, USA) was used. A releasing back incision was made from the choana on the vertical palatine bone under the sphenopalatine artery (Figure 1a). The choana was outlined and the incision continued on the septum 2-3mm away from its posterior edge to ensure that the incision was down to bone at all times. The incision in the floor of the nose was brought forward at a variable distance laterally on the floor to near the inferior turbinate (Figure 1b). Then the superior incision started at the superior limit of the sphenoid ostium and the striated 'thin' upper septal mucosa is preserved. The superior septal mucosa was thin, making it less effective for reconstructive purposes, contains the olfactory epithelium and avoided if preservation of olfaction was intended. The anterior limit was usually to the muco-squamous junction in the nasal vestibule (Figure 1c). The flap was mobilized everywhere but superiorly. The release from the superior edge was made last. The flap was stored in the nasopharynx or maxillary sinus for later use in reconstruction (Figure 1d).

\section{Postoperative care}

Silastic sheeting $0.51 \mathrm{~mm}$ (Medtronic, Jacksonville, FL, USA) was used to cover the septum bilaterally. Mupiricon 2\% ointment and Amoxycillin $875 \mathrm{mg} /$ Clavulinic acid $125 \mathrm{mg}$ was used twice daily for 10 days. This was intended to reduce Staphylococcal coIonization in the immediate post-surgical period. Large volume, positive pressure nasal irrigation with commercially prepared buffered isotonic saline was used via a $240 \mathrm{ml}$ squeeze bottle (Sinus Rinse, Neilmed, CA, USA). This was continued twice daily for 3 weeks, at which the first post-operative outpatient review occurred. The silastic sheets were removed and saline irrigation continued with instructions for daily use decreasing to 2-3 times weekly, but not stopped until 90 days post-op, when the majority of healing had occurred ${ }^{(13)}$. All sinonasal cavities were examined between 3 and 6 months to check for remucosalization, the absence of crusting, recovery of mucillary function, and the absence of chronic inflammation (apart from occasional small areas of granulation tissue). This process was mostly completed by 3 months $^{(13)}$ (Figure 2).

\section{Statistical analysis}

Statistical analyses were performed using SPSS v 20.0 (Statistical Package for the Social Sciences, Chicago, IL, USA). Age, nasal symptom scores and SNOT22 data were considered to be parametric and the paired Student's t test was used to compare pre-op and post-op scores, and the independent samples t test was used for comparisons between study groups. Ordinal data from the nasal obstruction question and Global nasal function scores were assessed with a Kendal Tau-b for changes between study groups. Chi squared analysis of proportions was used for gender and all confounding factor assessment. All p-values were two tailed and a value of $p<0.05$ was considered statistically significant.

Table 1. Allocation table for baseline characteristics between groups.

\begin{tabular}{|lccc|}
\hline & $\begin{array}{c}\text { Nasoseptal } \\
\text { flap }\end{array}$ & No flap & p value \\
\hline $\mathrm{n}$ & 42 & 76 & \\
\hline Age (yrs) & $55.52 \pm 18.35$ & $54.37 \pm 17.25$ & 0.74 \\
\hline Gender (\% female) & $40.5 \%$ & $39.5 \%$ & 0.92 \\
\hline Draf 3 performed & $23.8 \%$ & $14.7 \%$ & 0.22 \\
\hline Dura resection & $88.1 \%$ & $24.3 \%$ & $<0.01^{*}$ \\
\hline $\begin{array}{l}\text { Olfactory bulb and tract } \\
\text { removed }\end{array}$ & $40.5 \%$ & $17.1 \%$ & $<0.01^{*}$ \\
\hline Radiotherapy & $33.3 \%$ & $12.9 \%$ & $0.01^{*}$ \\
\hline Neoplasm (\%malignant) & $38.1 \%$ & $31.6 \%$ & 0.47
\end{tabular}



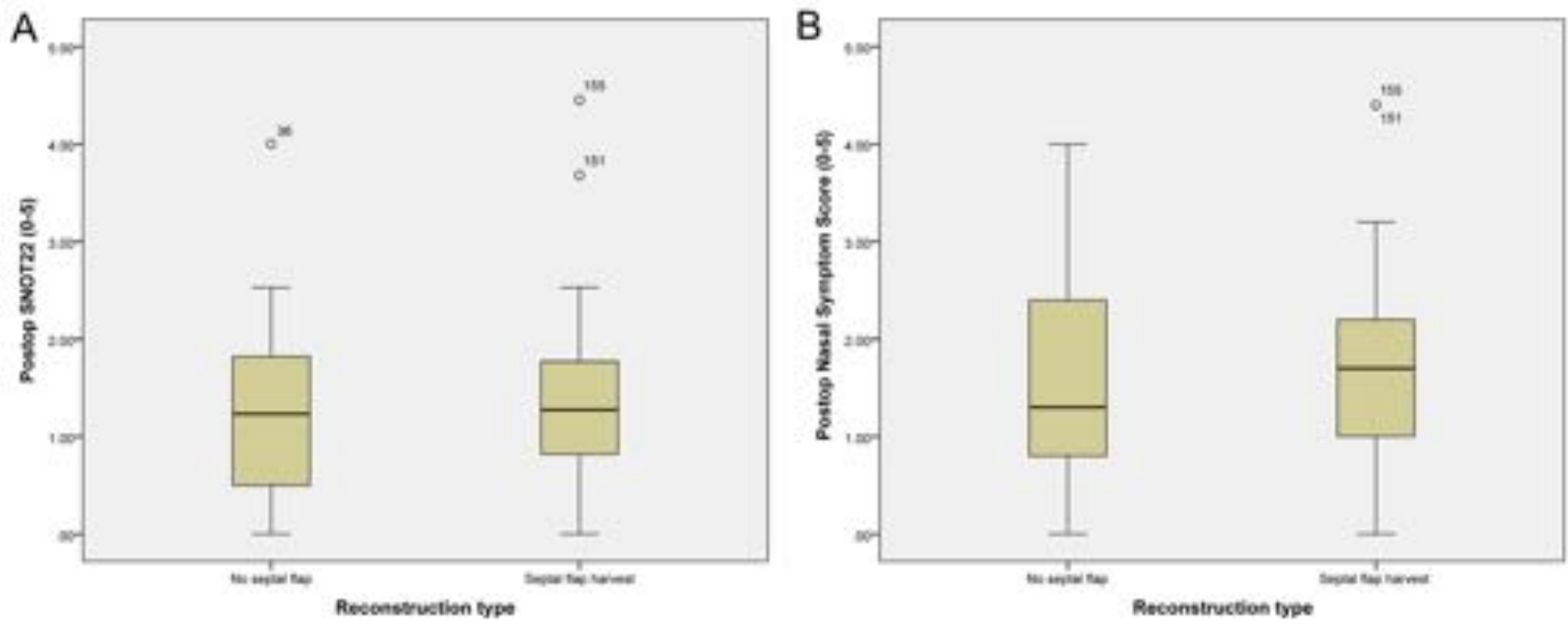

Figure 3. The impact of the nasoseptal flap on disease specific quality of life (A) and nasal symptom scores (B) in patients undergoing endoscopic resection of neoplasia.

\section{Results}

One hundred and eighteen (118) patients ( $40 \%$ female, age 55 \pm 18 years) were recruited. Patient baseline characteristics of the study groups are described in Table 1 . As expected, the dural resection and olfactory apparatus removal were over represented in the nasoseptal flap group. Similarly, radiotherapy was more commonly given to the nasoseptal flap group. Mean followup was $13.00 \pm 10.1 \mathrm{mths}$. The list of pathologies managed is presented in Table 2 with malignant tumours accounting for $33.8 \%$ of surgeries performed. The baseline SNOT22 and Nasal symptoms scores were $1.36 \pm 0.87$ and $1.44 \pm 1.12$, respectively and compared similarly at follow-up (1.28 $\pm 0.90, p=0.93$ and $1.59 \pm 1.12, p=0.41)$.

\section{The impact of the nasoseptal flap}

There was no statistically significant difference in sino-nasal function at follow-up between patients who had a nasoseptal flap versus those who did not based on either the SNOT22 (1.33 \pm 0.98 v $1.23 \pm 0.85, p=0.65$ ) or the Nasal Symptom Score (1.71 $\pm 1.15 \vee 1.48 \pm 1.11, p=0.36$ ) (Figure 3 ). The overall rating of global nasal function was also similar between groups (nasoseptal flap group score 2.0(IQR4.0) v no nasoseptal flap group score 3.0(IQR5.0), $p=0.30$ ). There was an association between the use of nasospetal flap and sense of nasal obstruction, with less nasal obstruction reported in the nasoseptal flap group (1.0(IQR2.0) v 1.0(IQR3.0), $p=0.01$ Kendal's tau b).

\section{The impact of radiotherapy}

The most significant impact on post-operative nasal function was the adjuvant use of radiotherapy. The post-operative SNOT22 scores were higher $(1.73 \pm 0.96 \vee 1.15 \pm 0.84, p=0.01)$ and higher Nasal Symptom Scores $(2.30 \pm 1.02$ v $1.37 \pm 1.08$, $\mathrm{p}=0.002$ ) were reported in those with adjuvant radiotherapy versus those without. Global rating of nasal function was similar (2(IQR3.3) v 3(IQR4.0), p = 0.83, Kendal's Tau b). The difference in nasal obstruction scores approached statistical significance (Radiotherapy $1.5($ IQR2.0) v none $1.0(2.0), p=0.06$, Kendal's tau $b$ )

Impact of including a Draf $\mathbf{3}$ or modified Lothrop as part of the exposure

No negative influence could be seen from the inclusion of a Draf 3 in the approach. There was no statistically significant diffe-

Table 2. Pathology casemix for the cohort.

\begin{tabular}{|c|c|c|}
\hline Pathology & $\mathbf{n}$ & $\%$ \\
\hline Benign paranasal (other) & 33 & 28.0 \\
\hline Meningioma & 22 & 18.6 \\
\hline Papilloma & 15 & 12.7 \\
\hline Minor Salivary Carcinoma & 13 & 11.0 \\
\hline Craniopharyngioma/Cyst & 10 & 8.5 \\
\hline Olfactory Neuroblastoma & 8 & 6.8 \\
\hline SCC & 7 & 5.9 \\
\hline Malignant paranasal (other) & 5 & 4.2 \\
\hline Chordoma & 3 & 2.5 \\
\hline Epidermoid & 2 & 1.7 \\
\hline Total & 118 & 100.0 \\
\hline
\end{tabular}



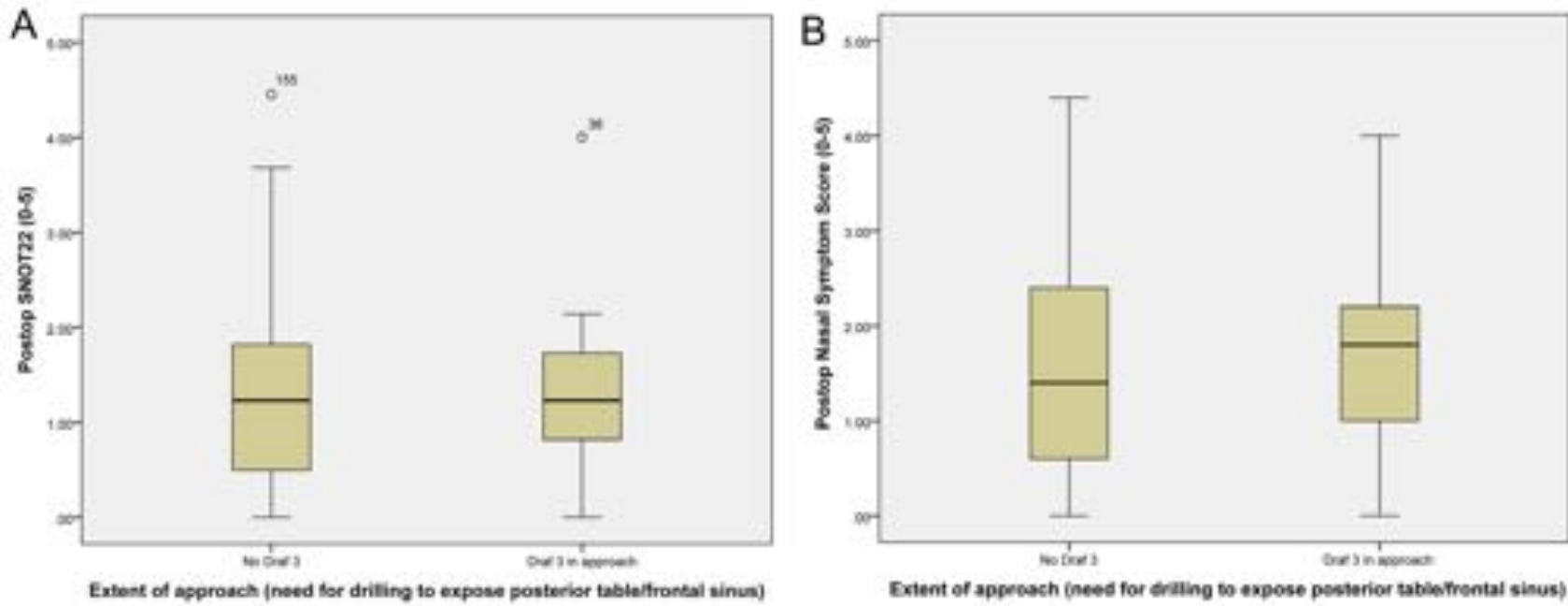

Exient of appecach (need for traling to expose pesterior tabienthental sinus)

Figure 4. Radical sinus procedures that modify the normal anatomy to gain access such as the Draf 3 had no impact on disease specific quality of life (A) and nasal symptom scores (B) in patients undergoing endoscopic resection of neoplasia.

rence in SNOT22 scores in patients who had a Draf 3 approach (1.34 \pm 0.90 v $1.26 \pm 0.90, p=0.75)$, and Nasal Symptom Scores were not significantly different $(1.76 \pm 1.01 \vee 1.52 \pm 1.16, p=$ 0.44 ) (Figure 4). Both Global rating of nasal function and sense of nasal obstruction were unaffected (2.0(IQR4.0) v 3.0(IQR4.0), $\mathrm{p}=$ 0.33 Kendals Tau b) and (1.0(IQR2.0) v 1.0(IQR 3.0), Kendals Tau b, $p=0.92)$.

\section{The influence of olfactory apparatus removal}

On initial analysis, the removal of the olfactory apparatus appeared to have a significant impact on SNOT22 scores (1.73 \pm 0.98 v $1.10 \pm 0.80, p=0.002)$ and Nasal Symptom Score (2.18 \pm 0.98 v $1.33 \pm 1.10, p=0.003$ ). However, as "olfaction" makes up a component of these scores, a sensitivity analysis was performed with the 'olfaction' question removed from the nasal symptom score $(1.74 \pm 1.03 \vee 1.32 \pm 1.16, p=0.13)$, and with the psychosocial domain of the SNOT22 compared in isolation $(1.49 \pm$ 0.96 v $1.13 \pm 0.83, p=0.08$ ), revealing no significant difference in non-olfactory sinonasal symptoms. Global ratings of nasal function were (2.0(IQR4.0) v 3.0(IQR4.0), $\mathrm{p}=0.51)$.

Dural resection had little impact on postop function as assessed by SNOT22 (1.39 \pm 0.98 v $1.17 \pm 0.82, p=0.28)$, Nasal Symptoms Score $(1.77 \pm 1.12 \vee 1.39 \pm 1.12, p=0.12)$, and global nasal function score (2.0(IQR5.0) v 3.0(4.0), $p=0.86$ Kendal's Tau b). However, having a malignant tumour had a negative impact on aspects of postop function compared to benign neoplasia: SNOT22 $(1.79 \pm 0.80 \vee 1.01 \pm 0.84, \mathrm{p}<0.01)$, NSS $(2.26 \pm 0.98$ v $1.21 \pm 1.03, \mathrm{p}<0.01)$ and Nasal Obstruction (2.0(IQR2.0) v 1.0(IQR2.0), $\mathrm{p}<0.01)$. However, Global function score was not significantly different (2.0(IQR3.5) v3.0(IQR4.0), p = 0.56). This sub-analysis includes the influence of a range of surgical and treatment factors that results in this outcome (Figure 5).

\section{Discussion}

Comparing the sinonasal function of patients before and after their treatment has little external validity if the groups are fundamentally different, such as a comparison of paranasal sinus tumors with skull base tumors. Such a comparison fails to take into account the fact that some patients have extensive pre-treatment sinonasal dysfunction from pathology within the paranasal sinuses while others have near normal sinonasal tracts and their pathology primarily involves the skull base or intradural structures. However, assessing the impact of surgical technique and adjuvant therapies on the subsequent sinonasal cavity and function is of value. The data presented in this study suggests that most patients have good sinonasal function postoperatively with $71.1 \%$ of patients rating their function above the neutral (0) score. The post treatment sinonasal function was not influenced by surgical techniques, nasoseptal flap or use of the Draf 3, but was influenced by factors that contributed to loss of function (olfactory loss and post-operative radiotherapy). Radiotherapy has only been recently reported as an important factor in determining outcome following endoscopic sinonasal tumour management ${ }^{(10)}$. There is, however, much literature on the influence of radiotherapy, with or without chemotherapy, in patients with nasopharyngeal tumours ${ }^{(14)}$. Castelnuovo et al. demonstrated that QoL scores were worse in those $>60 \mathrm{yrs}$ of age, who had radiotherapy or those needing craniectomy to remove extensive tumours ${ }^{\left({ }^{(10)}\right.}$. Palme et al. also demonstrated that radiotherapy was the major factor influencing quality of life for patients with anterior skull base neoplasms treated with both open and endoscopic approaches ${ }^{(11)}$. From the literature on the management of nasopharyngeal carcinoma, radiotherapy can have effects on both local function ${ }^{(15)}$ and cognitive function ${ }^{(16)}$. Long term adverse effects of radiation to the skull base such as 

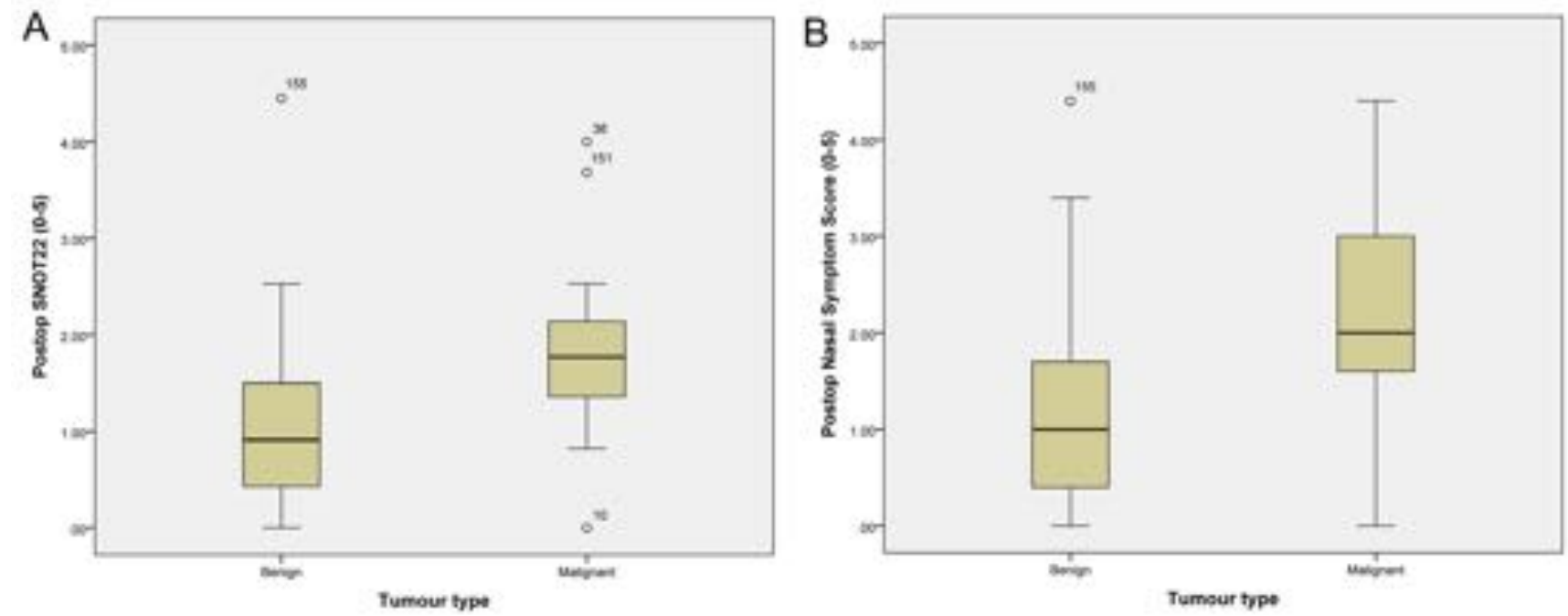

Figure 5. The presence of a malignant pathology was a predictor for poor performance specific quality of life (A) and nasal symptom scores (B) in patients undergoing endoscopic resection of neoplasia due to multiple factors.

endocrinopathy ${ }^{(17)}$, radionecrosis ${ }^{(18)}$, cranial neuropathy ${ }^{(19)}$ and hearing impairment ${ }^{(20)}$ were not addressed here. With only 13 months follow-up, and from personal observation, it is conceivable that although some of the local mucosal post-radiation effects may have resolved, late radiation adverse effects can still occur. Late complications from radiotherapy are uncommon $(6 \%)^{(20)}$. Lin et al. showed that they mostly occur at a mean duration to event $5.4 \pm 4.4$ years with a latency range from 1-20years (19).

There have been several studies looking at the local sinonasal function and olfaction after endoscopic interventions uitlizing the nasoseptal flap ${ }^{(6,7,21)}$. However, many of these studies compare mismatched groups with disparate numbers of patients with more extensive skull base disease in the NSF group ${ }^{(7,21)}$. The decision to take a more expanded approach through otherwise unaffected sinuses is balanced against the morbidity associated with brain retraction ${ }^{(8)}$. Also, with limited information about specific surgical technique in several studies, some of the olfactory consequences may have been related to unintentional damage to the 'olfactory strip' when creating the NSF or performing the septectomy ${ }^{(22,23)}$. The data presented in this study demonstrates the influence of surgical technique on sinonasal function in comparable patient populations regardless of their degree of pre-operative sinonasal morbidity.

Despite more extensive resection of the dura, destruction of the olfactory bulb and post-operative radiotherapy in the nasoseptal flap group, the sinonasal performance was similar to the group without a nasoseptal flap. This favourable outcome in the NSF group is further strengthened when the baseline data potentially biases better function to the non-NSF group. This series suggests that the use of a NSF for extensive skull base resections has little negative impact on the subsequent function of the sinonasal tract. Additionally, nasal obstruction scores were lower in the nasoseptal flap group. Nasal obstruction was uncommon in the population, as a whole, with only $14.1 \%$ of respondents noting it as a moderate problem or worse. In the senior authors' experience, when patients complain of nasal obstruction, there is usually a cause, such as local mucositis in the vestibule or post-operative formation of adhesions. Obstruction is not simply due to loss and then re-mucosalization of the septum although this process should not be under-estimated. There are techniques to reduce this time to remucosalization such as silastic covering ${ }^{(24)}$, free grafting ${ }^{(25)}$ or the "reverse flap" technique ${ }^{(26)}$. However, as with radiotherapy, the permanent impact of re-mucosalization of the septum is more reliably assessed after 3 months ${ }^{(13)}$.

\section{Conclusions}

The creation and utilization of a NSF after endonasal skull base resections does not appear to affect the quality of life and sinonasal function of patients when compared to a similar group of patients who did not have a NSF. Interestingly, loss of function from adjuvant therapy or the need to resect functional areas, such as the olfactory apparatus did have a negative impact.

\section{Author contributions}

$\mathrm{RH}$ : design, data collection, manuscript preparation, statistical analysis; JM: data collection; MW: expert review and data contribution; AD: expert review and data contribution; TS: expert review and data contribution; NM: data collection; HB: expert review, data collection and analysis; AK: expert review, data collection and analysis; CT: expert review and data contribution. 


\section{Conflict of interest}

Richard J. Harvey, has served on an advisory board for Schering Plough and Glaxo-Smith-Kline, previous consultant with Medtronic, Olympus and Stallergenes, speakers' bureau for Merek Sharp Dolme and Arthrocare and has received grant support from NeliMed. Charles Teo is a consultant for Aesculap. Mark Winder, Andrew Davidson, Tim Steel, Henry Barham and Anna Knisley have no financial disclosures and no conflict of interest.

\section{References}

1. Harvey RJ, Parmar P, Sacks R, Zanation AM Endoscopic skull base reconstruction of large dural defects: a systematic review of published evidence. Laryngoscope. 2012 122: 452-459.

2. Harvey RJ, Smith JE, Wise SK, Patel SJ, Frankel BM, Schlosser RJ. Intracranial complications before and after endoscopic skull base reconstruction. Am J Rhinol. 2008; 22: 516-521.

3. Harvey RJ, Winder M, Davidson A, Steel T, Knisely A, Barham $\mathrm{H}$, et al. Collagen matrix underlay for endoscopic skull base reconstruction. Manuscript in submission.

4. Solyar AY, Fried MP, Goldberg AN, Kennedy DW, Lanza DC. Pedicled nasoseptal flap is not the standard of care for skull base defects. Laryngoscope. 2011; 121: 896-897.

5. Zanation AM, Thorp BD, Parmar P, Harvey RJ. Reconstructive options for endoscopic skull base surgery. Otolaryngol Clin North Am. 2011; 44: 1201-1222.

6. Tam S, Duggal N, Rotenberg BW. Olfactory outcomes following endoscopic pituitary surgery with or without septal flap reconstruction: a randomized controlled trial. Int Forum Allergy Rhinol. 2013; 3: 62-65.

7. Alobid I, Ensenat J, Marino-Sanchez F, Rioja E, de Notaris M, Mullol J, et al. Expanded endonasal approach using vascularized septal flap reconstruction for skull base tumors has a negative impact on sinonasal symptoms and quality of life. Am J Rhinol Allergy. 2013; 27: 426-431.

8. de Almeida JR, Carvalo F, Vaz Guimaraez Filho F, Koutourousiou M, Su SY, Wang E, et al. A comparison of post-operative MRI signal changes between endoscopic endonasal approaches and open approaches for olfactory groove meningiomas: a match paired analysis from two institutions. J Neurol Surg B 2014; 75 - A048.

9. Chin D, Snidvongs K, Kalish L, Sacks R, Harvey RJ. The outside-in approach to the modified endoscopic Lothrop procedure. Laryngoscope. 2012; 122: 1661-1669.

10. Castelnuovo P, Lepera D, Turri-Zanoni M Battaglia P, Bolzoni Villaret A, Bignami M, et al. Quality of life following endoscopic endonasal resection of anterior skull base cancers. J Neurosurg. 2013; 119: 1401-1409.

11. Palme CE, Irish JC, Gullane PJ, Katz MR, Devins GM, Bachar G. Quality of life analysis in patients with anterior skull base neoplasms. Head Neck. 2009; 31: 1326-1334.

12. Hopkins C, Gillett S, Slack R, Lund VJ, Browne JP. Psychometric validity of the 22-item Sinonasal Outcome Test. Clin Otolaryngol. 2009; 34: 447-454.

13. Jo HW, Dalgorf DM, Snidvongs K, Sacks $R$, Harvey RJ. Postoperative irrigation therapy after sinonasal tumor surgery. Am J Rhinol Allergy. 2014; 28: 169-171.

14. Fang F-M, Tsai W-L, Lee T-F, Liao K-C, Chen $\mathrm{H}-\mathrm{C}, \mathrm{Hsu} \mathrm{H}-\mathrm{C}$. Multivariate analysis of quality of life outcome for nasopharyngeal carcinoma patients after treatment. Radiother Oncol. 2010; 97: 263-269.

15. Yin G-D, Xiong G-X, Zhao C, Chen Y-Y. Damage of nasal mucociliary movement after intensity-modulated radiation therapy of nasopharyngeal carcinoma. Chin. 2010; 29: 824-829.

16. Tang $Y$, Luo D, Rong $X$, Shi $X$, Peng $Y$. Psychological disorders, cognitive dysfunction and quality of life in nasopharyngeal carcinoma patients with radiation-induced brain injury. PLoS ONE. 2012; 7(6):e36529.

17. Lue B-H, Huang T-S, Chen H-J. Physical distress, emotional status, and quality of life in patients with nasopharyngeal cancer complicated by post-radiotherapy endocrinopathy. Int J Radiat Oncol Biol Phys. 2008; 70: 28-34.

18. Hua $\mathrm{Y}-\mathrm{J}$, Chen M-Y, Qian C-N, Hong M-H, Zhao C, Guo L, et al. Postradiation nasopharyngeal necrosis in the patients with nasopharyngeal carcinoma. Head Neck. 2009; 31: 807-812.

19. Lin Y-S, Jen Y-M, Lin J-C. Radiation-related cranial nerve palsy in patients with nasopharyngeal carcinoma. Cancer. 2002; 95: 404-409.

20. Sumitsawan Y, Chaiyasate S, Chitapanarux I, Anansuthiwara M, Roongrotwattanasiri K, Vaseenon V, et al. Late complications of radiotherapy for nasopharyngeal carcinoma. Auris Nasus Larynx. 2009; 36: 205-209.

21. Alobid I, Ensenat J, Marino-Sanchez F de Notaris M, Centellas S, Mullol J, et al. Impairment of olfaction and mucociliary clearance after expanded endonasal approach using vascularized septal flap reconstruction for skull base tumors. Neurosurgery. 2013; 72: 540-546.

22. Simal Julian JA, Miranda Lloret P, Pancucci G, Sanroman Alvarez P, Botella Asuncion C. Avoiding olfactory impairment after endoscopic endonasal expanded approaches. Neurosurgery. 2013; 73: E562-563.

23. Harvey RJ, Winder M, Davidson A, Steel T, Nalavenkata S, Mrad N, et al. The olfactory strip and its preservation with a modified nasoseptal flap in endoscopic pituitary surgery maintains smell and sinonasal function. Manuscript in submission.

24. Chin D, Harvey RJ. Frontal, Cribiform and Ethmoid Roof Defects. In: Bleier B, editor. Comprehensive Techniques in CSF Leak and Skull Base Reconstruction. Basel, Switzerland Karger AG - Medical and Scientific Publishers 2012.

25. Kimple AJ, Leight WD, Wheless SA, Zanation AM. Reducing nasal morbidity after skull base reconstruction with the nasoseptal flap: free middle turbinate mucosal grafts. Laryngoscope. 2012; 122: 1920-1924.

26. Caicedo-Granados E, Carrau R, Snyderman $\mathrm{CH}$, Prevedello D, Fernandez-Miranda J, Gardner $\mathrm{P}$, et al. Reverse rotation flap for reconstruction of donor site after vascular pedicled nasoseptal flap in skull base surgery. Laryngoscope. 2010; 120: 1550-1552.

Richard Harvey

Ground Floor

67 Burton Street

Darlinghurst NSW 2010

Australia

Tel: +61 (02) 93604811

Fax: +61 (02) 93609919

E-mail: richard@sydneyentclinic.com 